$4 \quad$ ANTERIOR QUADRATUS LUMBORUM BLOCK FOR POSTOPERATIVE RECOVERY AFTER TOTAL HIP ARTHROPLASTY: A RANDOMIZED, MULTIPLE-BLIND, PLACEBO-CONTROLLED TRIAL

M Kikuchi* ${ }^{*}$ Y Mizuno, T Goto. Yokohama City University Hospital, Yokohama, Japan

\subsection{6/rapm-2021-ESRA.4}

Background and Aims Appropriate pain management is essential to improve the postoperative recovery after total hip arthroplasty (THA). Some randomized trials have indicated that anterior quadratus lumborum block (QLB) provides effective postoperative analgesia in THA. However, whether anterior QLB improves postoperative recovery after THA is unclear.

Methods The participants were randomly assigned to either the anterior QLB or placebo groups. After induction of general anesthesia, anterior QLB was performed by using $0.25 \%$ levobupivacaine or normal saline. The primary outcome was the quality of recovery 40 score (QoR-40). Secondary outcomes included the visual analog scale score of pain intensity at rest and movement, intraoperative and postoperative doses of fentanyl, and incidence of postoperative nausea and vomiting.

Results This study included and analyzed 70 participants of the anterior QLB group and 69 participants of the placebo group. The pain dimension in QoR-40 score 24 hours after the surgery was higher in the anterior QLB group than in the placebo group (median 30.5 [IQR 27.0, 32.0] vs 28.0 [24.0, 32.0] $\mathrm{p}=0.033)$. However, total score of QoR-40, which is the primary outcome, were not statistically significant different between each group (169 [153, 177] vs 158 [142, 177] $\mathrm{p}=0.122$ ) (table 1). The anterior QLB group needed less intraoperative dose of fentanyl than the placebo group (275 $[200,350]$ vs $350[250,425] \mathrm{p}=0.007)$. Other secondary outcomes were not statistically significant different.

\begin{tabular}{|c|c|c|c|}
\hline & Anterfor CLB(n-70) & Placebo(n=69) & P value \\
\hline Total score of QOR-40 & $168.50[153.25,177.00]$ & $158.00[142.00,177.00]$ & 0.122 \\
\hline - physical comfort & $50.50[45.25,54.00]$ & $47.00[41.00,54.00]$ & 0.156 \\
\hline - emotional state & $39.00[35.25,41.00]$ & $38.00[33.00,42.00]$ & 0.483 \\
\hline - physical independence & $15.50[12.00,20.00]$ & $15.00[12.00,19.00]$ & 0.886 \\
\hline - psychological support & $34.00[29.00,35.00]$ & $33.00[29.00,35.00]$ & 0.525 \\
\hline - pain & $30.50[27.00,32.00]$ & $28.00[24.00,32.00]$ & 0.033 \\
\hline
\end{tabular}

Conclusions Anterior QLB combined with general anesthesia did not improved postoperative recovery after total hip arthroplasty.

\section{COMPARISON OF PERIPHERAL NERVE BLOCK WITH GENERAL ANESTHESIA AND GENERAL ANESTHESIA ALONE IN TERMS OF POSTOPERATIVE DELIRIUM AND COMPLICATIONS USING A NATIONWIDE DATABASE}

M Yoshimura*, H Shiramoto, M Koga, A Yoshimatsu, Y Morimoto. Ube Industries Central Hosp., Ube, Japan

10.1136/rapm-2021-ESRA.5

Background and Aims The effect of peripheral nerve block (PNB) under general anesthesia (GA) on the clinical outcome comparing GA alone remains unknown. We hypothesized that $\mathrm{PNB}$ is associated with reduced postoperative delirium and improved patients morbidity after surgical procedures.

Methods We used a nationwide inpatient database in Japan to compare patient outcomes by GA with PNB versus GA alone from April 2016 to October 2019. Our primary outcome was postoperative delirium. The incidence of morbidity were secondary outcomes. We conducted propensity score matched analyses of patients who underwent all surgical procedures using 41 covariates. Chi-square analyses were performed to calculate odds ratios and their 95\% confidence intervals (CI). For sensitivity analyses, we performed instrumental variables and restricted the definition of postoperative delirium and subgroup.

Results Of 591,578 patients, 82,461 received GA-PNB, and 509,117 received GA group. After one to four propensity score matching, 81,873 patients were included in the GA-PNB group and 204,932 in the GA group. The adjusted odds ratios for postoperative delirium, composite morbidity were 0.953 (95\%CI 0.924 to 0.982 ), 0.766 (95\%CI 0.727 to 0.806 ), respectively, for the GA-PNB group with reference to the GA group. For sensitivity analyses, findings were also consistent with instrumental variable and subgroup analyses.

Conclusions This retrospective, nationwide cohort study demonstrated that PNB underwent GA was associated with reduced postoperative delirium and composite morbidity.

\section{Best free paper session II - Chronic pain}

\section{REAL-TIME ULTRASOUND-COMPUTED TOMOGRAPHY IMAGE FUSION TRANSFORAMINAL LUMBAR APPROACH}

${ }^{1}$ A Pourveur* , ${ }^{2} \mathrm{~J}$ Gouwy, ${ }^{2} \mathrm{M}$ Renard, ${ }^{2} \mathrm{I}$ Mocanu, ${ }^{2} \mathrm{~S}$ Pather, ${ }^{2} \mathrm{O}$ Vannieuwenhove, ${ }^{1} \mathrm{E}$ Guntz. 'Université Libre de Bruxelles/CHIREC de Braine I'Alleud-Waterloo, Braine-l'Alleud, Belgium; ${ }^{2}$ CHIREC de Braine I'Alleud-Waterloo, Braine-l'Alleud, Belgium

\subsection{6/rapm-2021-ESRA.6}

Background and Aims Transforaminal approach under ultrasounds (US) remains challenging. We evaluated on phantoms if fusions of computed tomography (CT) images with dynamic US examinations lead to precise location and puncture of the foramina between the fourth and fifth lumbar vertebra (L4L5).

Methods Three anesthesiologists performed fusions of US and CT images with 3 different techniques on 2 models of phantom. Technique 1: fusion of the edge of the spinous process of L5 and the 2 posterior superior iliac spines. Technique 2: location of the 2 lateral extremities of the laminae instead of iliac spines. Technique 3: skin landmarks. Comparisons were performed with the value of precision (VP). 3 punctures targeting the right L4-L5 foramina were performed, needles positions were checked under X-ray. VPs were compared with ANOVAs, $\mathrm{p}<0.05$ considered significant and results reported as means \pm standard deviation.

Results One hundred and fifty fusions were recorded. Techniques 1 and 2 were performed on the gelatin phantom; technique 2 was superior to technique 1 (VP: $1.12 \pm 0.54$ vs 2.38 \pm 1.49 for operator $1,0.6 \pm 0.39$ vs $3.66 \pm 1.22$ for operator $2,0.89 \pm 0.31$ vs $1.23 \pm 0.63$ for operator $3, \mathrm{p}<0.001)$. There was no difference between the 3 techniques evaluated with the marketed phantom. X-ray examinations confirmed 